\title{
Effects of Chain Length on the Immunogenicity in Rabbits of Group B Streptococcus Type III Oligosaccharide-Tetanus Toxoid Conjugates
}

\author{
Lawrence C. Paoletti, “ Dennis L. Kasper, ${ }^{\star *}$ Francis Michon, José Difabio, ${ }^{5}$ Harold J. Jennings," \\ Tor D. Tosteson, ${ }^{*}$ and Michael R. Wessels** \\ *Channing Laboratory, Brigham and Women’s Hospital, and ${ }^{\ddagger}$ Division of Infectious Diseases, Beth Israel Hospital, \\ Harvard Medical School, Boston, Massachusetts 02115; and ${ }^{\S}$ Division of Biological Sciences, \\ National Research Council of Canada, Ottawa, Ontario, K1A OR6 Canada
}

\begin{abstract}
One method to improve the immunogenicity of polysaccharide antigens is the covalent coupling of the native polysaccharide or a derivative oligosaccharide to a carrier protein. In general, $\mathbf{T}$ cell-dependent properties are enhanced in conjugates of smaller saccharides, but a conformational epitope of the native polysaccharide may be better expressed in conjugates of larger saccharides. We have reported previously the synthesis and immunogenicity in animals of an oligosaccharide-tetanus toxoid conjugate vaccine against type III group B Streptococcus. In this study, we sought to determine the optimal size of group B Streptococcus type III oligosaccharide for use in a conjugate vaccine by evaluating the relative immunogenicity of conjugate vaccines containing oligosaccharides that were twofold smaller $\left(7,000 M_{\mathrm{r}}\right)$ or larger $\left(27,000 M_{\mathrm{r}}\right)$ than that reported previously $\left(14,500 M_{r}\right)$. All three type III oligosaccharide conjugate vaccines were immunogenic in rabbits, in contrast to native, uncoupled group B Streptococcus type III polysaccharide. However, with respect to eliciting specific antibodies that were protective in vivo, the vaccine containing the intermediate-size oligosaccharide was superior to the smaller or larger conjugate vaccine. Analysis of opsonic activity of vaccine-induced antibodies demonstrated a predominance of IgG antibodies, thought to reflect $T$ cell dependence, in response to shorter chain length conjugates, while the conformational epitope of the native polysaccharide was maximally expressed on longer chain length conjugates. These opposing trends may account for the optimal immunogenicity of an intermediate-size group B Streptococcus type III oligosaccharide conjugate vaccine. (J. Clin. Invest. 1992. 89:203-209.) Key words: neoglycoconjugate • sialic acid • polysaccharide $\bullet$ Streptococcus agalactiae $\bullet$ conformational epitope
\end{abstract}

\section{Introduction}

Coupling of polysaccharides or derivative oligosaccharide fragments to protein carriers has been an effective approach to enhancement of the immunogenicity of a variety of polysaccha-

This work is issued as National Research Council of Canada No. 31975.

Address correspondence to Lawrence C. Paoletti, Channing Laboratory, 180 Longwood Ave., Boston, MA 02115.

Received for publication 25 February 1991 and in revised form 17 June 1991.

J. Clin. Invest.

(c) The American Society for Clinical Investigation, Inc.

0021-9738/92/01/0203/07 \$2.00

Volume 89, January 1992, 203-209 ride antigens (1-10). The mechanisms by which coupling of a polysaccharide or derivative oligosaccharide to a carrier protein results in enhanced immunogenicity are not well defined but are thought to involve the recruitment of $\mathrm{T}$ helper cells. Conjugation to a protein may alter the relatively $\mathrm{T}$ cell-independent immune response to polysaccharides to one with characteristics of the response to a $T$ cell-dependent immunogen. Such $T$ cell-dependent characteristics include increased antibody response (predominantly of the IgG class) and augmentation of specific antibody levels after booster vaccinations.

Two opposing considerations relate directly to optimizing the size of the saccharide in a conjugate vaccine: $(a)$ Theoretical considerations and limited experimental evidence suggest that saccharide fragments of shorter chain length, as opposed to high-molecular-weight polysaccharides, may be better able to elicit $\mathrm{T}$ cell-dependent antibody responses. A relatively shortchain-length oligosaccharide, as part of a conjugate vaccine, may optimize $T$ cell help by bringing antigen-specific $B$ cells and helper $\mathrm{T}$ cells into a more favorable physical relationship for cell-cell interaction. (b) However, the size of the haptenic saccharide must be sufficiently large to express the complete epitope of the native antigen. For certain bacterial capsular polysaccharides, there is increasing evidence that the epitope recognized by protective anticapsular antibodies is a conformational one, fully expressed only in longer-chain-length saccharides. This phenomenon has been demonstrated for antibody binding to the group B meningococcal polysaccharide $(11,12)$, group B Streptococcus (GBS) type III polysaccharide (13), and the type 14 pneumococcal polysaccharide (14). Kabat et al. (15) found similar evidence of a chain length-dependent conformational epitope in studies with a monoclonal antibody directed to the Escherichia coli K1-group B meningococcal polysialic acid capsule. Thus, a longer chain length may express more fully the native epitope of the polysaccharide and therefore may be preferable, in terms of epitope expression, as part of a conjugate vaccine.

We have reported that covalent attachment of a $14,500 M_{\mathrm{r}}$ oligosaccharide ${ }^{1}$ fragment of the GBS type III polysaccharide to tetanus toxoid (TT) ${ }^{2}$ dramatically increased immunogenic-

1. The term "oligosaccharide" is generally used to designate compounds containing between 2 and 10 monosaccharide units per molecule (27). For convenience and consistency with related published material $(1,10,13,26)$, the term "oligosaccharide" will be used in this paper to designate a polysaccharide fragment smaller than the native polysaccharide.

2. Abbreviations used in this paper: GBS, group B Streptococcus; IIIN, GBS type III native polysaccharide; TT, tetanus toxoid; ru, pentasaccharide repeating unit(s); 6-TT, 6-ru oligosaccharide-TT conjugate vaccine; 14-TT, 14-ru oligosaccharide-TT conjugate vaccine; $25-\mathrm{TT}$ 25-ru oligosaccharide-TT conjugate vaccine. 
ity in animals (7). Whereas our studies of that conjugate demonstrated improved immunogenicity as compared with that of the unconjugated native polysaccharide, they did not define the optimal chain length of such a conjugate. We now report a comparative immunogenicity study of model conjugates in which we employed oligosaccharides with chain lengths either one-half as long or twice as long as the 14 pentasaccharide repeating unit (ru) saccharide reported previously. Although some of the information regarding the 14-ru conjugate has been published (7), all experiments presented in this report were performed concurrently. Analysis of the functional activity of vaccine-induced antibodies indicated that $\mathrm{T}$ cell-dependent properties, reflected by the immunoglobulin isotype of opsonically active antibodies, are enhanced in short chain length conjugates, whereas expression of the conformational epitope of the polysaccharide is enhanced in long chain length conjugates. The results support the hypothesis that the ideal immunogen is an intermediate chain length conjugate, in which the combined effects of these opposing trends are optimized.

\section{Methods}

\section{Generation and purification of GBS type III oligosaccharides}

Capsular polysaccharide from GBS type III strain M781 was isolated and purified from culture supernatant fluids as described previously (9). Type III oligosaccharides were made by enzymatic depolymerization of the native polysaccharide with endo- $\beta$-galactosidase from Citrobacter freundii (7). Oligosaccharides were isolated from enzymatically digested type III polysaccharide by gel filtration on a Sephadex G-75 column $(1.6 \times 80 \mathrm{~cm})$ equilibrated with $10 \mathrm{mM}$ Tris buffer $(\mathrm{pH} \mathrm{7.0})$. The molecular size of individual oligosaccharides was estimated by calculation of $K_{\mathrm{av}}$ based on elution volumes of the 1-ru oligosaccharide $\left(M_{\mathrm{r}} 1,064\right)$, the 2-ru oligosaccharide $\left(M_{\mathrm{r}} 2,128\right)$ and T40 dextran $\left(M_{\mathrm{r}}\right.$ $40,000)$ standards. Column fractions with $K_{\mathrm{av}}$ values of 0.415 and 0.115 -corresponding to 6 and $25 \mathrm{ru}$, respectively-were recovered separately and lyophilized to dryness. The molecular size of each oligosaccharide pool was confirmed by repeat chromatography on the Sephadex G-75 column. The quantity of each oligosaccharide recovered was estimated by measurement of sialic acid content (16) and measurement of total hexose (17) with purified GBS type III polysaccharide used as a standard.

\section{Conjugation of type III oligosaccharides to tetanus toxoid}

Purified large (25 ru) and small (6 ru) type III oligosaccharides were linked via their reducing ends to purified TT (Institut Armand Frappier, Montreal) monomer with use of a 6-aminohexyl-1- $\beta$-D-galactopyranoside spacer molecule, as previously described for the 14-ru oligosaccharide (7). In brief, the aminoalkyl glycoside spacer was coupled to the 6-ru, 14-ru, or 25-ru type III oligosaccharide by reductive amination in the presence of $\mathrm{NaCNBH}_{3}$. After incubation at $37^{\circ} \mathrm{C}$ for $3 \mathrm{~d}$, the oligosaccharide-spacer adducts were purified on a Bio-Gel P2 column. The oligosaccharide-spacer adducts were then activated with galactose oxidase and conjugated to purified TT monomer by reductive amination with use of $\mathrm{NaCNBH}_{3}$. The conjugates were analyzed for carbohydrate content by the phenol-sulfuric acid method (17) with purified native type III polysaccharide used as a standard. Protein content was estimated by the method of Lowry et al. (18) as modified by Larson et al. (19), with BSA as a standard.

Immunization of animals with oligosaccharide-TT vaccines Groups of three New Zealand White female rabbits (Millbrook Farms, Amherst, MA), each weighing $\sim 2 \mathrm{~kg}$, were injected subcutaneously with $50 \mu \mathrm{g}$ of TT monomer, $50 \mu \mathrm{g}$ of 6-ru oligosaccharide-TT conjugate vaccine (6-TT), $50 \mu \mathrm{g}$ of 14-ru oligosaccharide-TT conjugate vaccine (14-TT, reference 7), $50 \mu \mathrm{g}$ of 25 -ru oligosaccharide-TT conjugate vaccine (25-TT), or $50 \mu \mathrm{g}$ of native type III GBS capsular polysaccharide (IIIN), each emulsified with complete Freund's adjuvant. All doses were calculated on the basis of total vaccine weight. Booster doses (50 $\mu \mathrm{g})$ of the same vaccine preparations, mixed with incomplete Freund's adjuvant, were given on days 20 and 41 . Serum was collected on days 0 , $20,34,41,55$, and 75 .

\section{ELISA}

Rabbit antibodies to type III GBS polysaccharide were quantified in an ELISA with goat anti-rabbit IgG conjugated to alkaline phosphatase (Tago Inc., Burlingame, CA) at a 1/3,000 dilution or goat anti-rabbit IgM (Sera-Lab Limited, Sussex, England) at a 1/500 dilution. Plates were coated with $100 \mathrm{ng}$ of poly(L-lysine)-type III polysaccharide per well as described previously (20). Titers of type III-specific IgG antibody were recorded as the reciprocal of the dilution that had an absorbance at $405 \mathrm{~nm}$ of $\geq 0.3$ when the reference serum (rabbit antiserum to whole GBS M732 cells used at a 1/80,000 dilution) reached an absorbance of 0.5 .

\section{ELISA inhibition}

The relative affinity of GBS type III-specific antibodies for type III saccharides of different chain lengths was assessed by competitive ELISA. Pooled rabbit antiserum $(75 \mu \mathrm{l})$ to each conjugate vaccine, diluted 20,000-fold, was added to an equal volume of inhibitor (6-ru, 14-ru, or 25-ru oligosaccharide or native type III polysaccharide). This mixture $(100 \mu \mathrm{l})$ was added to ELISA plates coated with poly(L-lysine)type III polysaccharide and incubated at $37^{\circ} \mathrm{C}$ for $1 \mathrm{~h}$. The secondary antibody used in this assay was goat anti-rabbit IgG conjugated to alkaline phosphatase (Tago Inc., Burlingame, CA) at a 1/3,000 dilution. The absorbance at $405 \mathrm{~nm}$ was recorded when the absorbance of wells containing uninhibited serum reached 0.5 . Inhibition binding curves were generated by serial fourfold dilution of the inhibitors. Results expressed as percentage inhibition were calculated as: $\%$ inhibition $=\left[\left(A_{405 n m}\right.\right.$ of uninhibited serum $-A_{405 \mathrm{~nm}}$ of inhibited serum $) / A_{405 \mathrm{~nm}}$ of uninhibited serum] $\times 100$.

\section{Isolation of IgG from immune rabbit serum}

IgG was separated from other immunoglobulin classes in immune serum by protein A affinity chromatography. Pooled serum $(0.5 \mathrm{ml})$ obtained on day 75 from rabbits vaccinated with oligosaccharide conjugates was passed through a protein A-agarose affinity column (Pierce Chemical Co., Rockford, IL) equilibrated with PBS (20 mM sodium phosphate, $150 \mathrm{mM} \mathrm{NaCl}, \mathrm{pH}$ 7.3). Serum components that eluted with the starting buffer were designated the IgM fraction, and those that eluted with $0.1 \mathrm{M}$ glycine (pH 3.0) were designated the IgG fraction. Each fraction was dialyzed overnight at $4^{\circ} \mathrm{C}$ against PBS and adjusted to a final volume of $5 \mathrm{ml}$ with PBS. Dilutions of each Ig fraction were normalized based on the original serum volume from which the fractions were purified. Separation of antibody class was confirmed by ELISA with goat anti-rabbit IgG ( $\gamma$ - and light-chain-specific) and IgM ( $\mu$-chain-specific; Sera-Lab, Westbury, NY) alkaline phosphatase conjugates.

\section{Opsonophagocytic assay}

To test the functional properties of vaccine-induced antibodies, we used an in vitro opsonophagocytic assay developed by Baltimore et al. (21). In brief, $0.05 \mathrm{ml}$ of pooled antiserum was mixed with $3 \times 10^{6}$ human peripheral blood leukocytes, $0.05 \mathrm{ml}$ of normal human serum previously absorbed with type III GBS organisms as a source of complement, and $1.5 \times 10^{6} \mathrm{CFU}$ of GBS type III strain M781 in a total volume of $0.5 \mathrm{ml}$ in modified Eagle's medium. Quantitative cultures were done at time 0 and after $60 \mathrm{~min}$ of incubation at $37^{\circ} \mathrm{C}$. Results expressed as the percentage of GBS cells killed were calculated as: \% killed $=[(C F U$ at $t=0 \mathrm{~min}-\mathrm{CFU}$ at $t=60 \mathrm{~min}) / \mathrm{CFU}$ at $t=0 \mathrm{~min}]$ $\times 100$. 


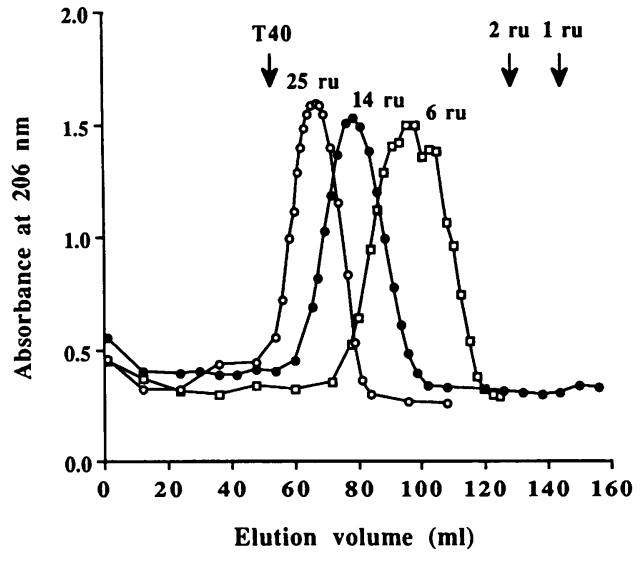

Figure 1. Composite of Sephadex G-75 elution profiles of size-fractionated group B Streptococcus type III oligosaccharides of 6 (6 ru), 14 (14 ru), and 25 (25 ru) pentasaccharide repeating units used for conjugation to tetanus toxoid. Arrows indicate position of molecular weight standards: 1 ru, GBS type III single-repeating-unit oligosaccharide $\left(M_{\mathrm{r}} 1,064\right) ; 2$ ru, GBS type III 2-repeating-unit oligosaccharide $\left(M_{\mathrm{r}} 2,127\right) ; \mathrm{T} 40, \mathrm{~T} 40$ dextran $\left(M_{\mathrm{r}} 40,000\right)$.

\section{Passive protection of mice by immune rabbit sera}

Pooled prevaccination or postvaccination rabbit serum (obtained on day 75$)$ was injected intraperitoneally $(0.2 \mathrm{ml}$ per mouse $)$ into 5 - to 6-wk-old Swiss-Webster female outbred mice (Harlan Sprague Dawley, Inc., Indianapolis, IN). $24 \mathrm{~h}$ later these mice were challenged with a lethal dose $\left(2.9 \times 10^{7} \mathrm{CFU}\right)$ of viable GBS strain M781 cells, which was administered intraperitoneally in a total volume of $1 \mathrm{ml}$ in ToddHewitt broth. Mice surviving the bacterial challenge were counted daily for $3 \mathrm{~d}$.

\section{Statistical analysis}

ELISA. The geometric mean endpoint titers for each group of animals were used to test for an increasing antibody response with increasing oligosaccharide size in the nonparametric Jonckheere-Terpstra test (StatXact version 1.0, Cytel Software Corp., Cambridge, MA).

ELISA inhibition. For the smallest (6-ru) and largest (IIIN) inhibitors a linear fit of the percent inhibition to the log of the inhibitor concentration was obtained with use of three inhibitor concentrations with the percentage inhibition closest to $50 \%$. These linear fits were used to estimate the ratio of the $\mathrm{IC}_{50}$ (i.e., the concentration of inhibitor that resulted in $50 \%$ inhibition of antibody binding to the native type
III polysaccharide-coated ELISA wells) for the smallest and largest inhibitors used. An 95\% confidence interval for the ratio was derived with standard errors obtained from the linear fit. A $\chi^{2}$ test was used to test for a trend in $\mathrm{IC}_{50}$ ratio among the three vaccination groups.

Passive protection of mice by immune rabbit sera. The log rank test was used to compare groups of mice with respect to length of survival following challenge with a lethal dose of GBS. Exact p-values were calculated with use of StatXact version 1.0.

\section{Results}

Synthesis of oligosaccharide-TT conjugates. Oligosaccharides were generated by enzymatic depolymerization of the native GBS type III polysaccharide with endo- $\beta$-galactosidase isolated from supernatant fluids of $C$. freundii cultures (7). Small (6 ru), medium (14 ru), and large ( $25 \mathrm{ru}$ ) oligosaccharides with average $M_{\mathrm{r}}$ values of 7,000 (range, 3,000-12,000), 14,500 (9,00019,000 ) and 27,000 (range, 16,500-38,000), respectively, were purified by gel filtration chromatography (Fig. 1). Synthesis and preliminary immunogenicity testing of the 14-ru oligosaccharide-TT conjugate vaccine was reported previously (7). Each oligosaccharide was coupled via the reducing end to a 6-carbon spacer molecule by reductive amination. The oligosaccharide-spacer adduct was then covalently conjugated to monomeric TT by reductive amination. Biochemical characteristics of the type III oligosaccharides and of the corresponding TT conjugates are presented in Table I. The dry-weight composition of 6-TT was 31\% carbohydrate and $69 \%$ protein; and that of 25-TT was $62 \%$ carbohydrate and $38 \%$ protein. The molar ratio of oligosaccharide to protein for 6-TT was 9.8 and that of 25-TT was 9.2 (Table I). These molar ratios are similar to the molar ratio of 7.8 for 14-TT (7).

Immunogenicity of type III oligosaccharide-TT vaccines. Because the oligosaccharide conjugates had similar molar ratios of carbohydrate to protein (Table I), we chose to administer the vaccines to rabbits on a basis of total weight. Normalization of vaccine dosage in this fashion affords direct comparison of the effect of oligosaccharide chain length on the immune response. Rabbits vaccinated with oligosaccharide-TT conjugates of all three chain lengths developed type III-specific antibodies, whereas animals receiving unconjugated type III polysaccharide or TT did not (Table II). ELISA showed only a slight rise in specific antibody elicited by the first dose of all three

Table I. Biochemical Analysis of Group B Streptococcus Type III Oligosaccharide-Tetanus Toxoid Conjugate Vaccines

\begin{tabular}{|c|c|c|c|}
\hline & 6-TT & $14-\mathrm{TT}^{*}$ & 25-TT \\
\hline \multicolumn{4}{|l|}{ Avg. no. of pentasaccharide } \\
\hline repeating units (range) $)^{\ddagger}$ & $6(3-11)$ & $14(8-18)$ & $25(16-36)$ \\
\hline Avg. mol wt (range) $)^{\ddagger}$ & $7,000(3,000-12,000)$ & $14,500(9,000-19,000)$ & $27,000(16,500-38,000)$ \\
\hline \multicolumn{4}{|c|}{ Amount of vaccine as tetanus toxoid } \\
\hline$(\% \text { of dry wt })^{\S}$ & 69 & 57 & 38 \\
\hline \multicolumn{4}{|c|}{ Amount of vaccine as carbohydrate } \\
\hline$(\% \text { of dry wt })^{\|}$ & 31 & 43 & 62 \\
\hline \multicolumn{4}{|c|}{ Mol oligosaccharide/mol tetanus } \\
\hline toxoid" & 9.8 & 7.8 & 9.2 \\
\hline
\end{tabular}

\footnotetext{
* The composition of 14-TT has been reported (7) and is presented here for convenience of comparison. ${ }^{*}$ Based on molecular weight of 1,063
} for GBS type III pentasaccharide repeating (13) unit and $K_{\mathrm{av}}$ values on Sephadex G-75. ${ }^{8}$ Protein content as measured by the method of Lowry et al. (18) using BSA as a standard. "Carbohydrate content determined by phenol-sulfuric acid assay (17) using native type III polysaccharide as a standard. 'Based on molecular weight of 150,000 for monomeric tetanus toxoid. 
Table II. Group B Streptococcus Type III Polysaccharide-specific Antibody Titers of Sera from Rabbits

Vaccinated with Unconjugated Native GBS Type III Polysaccharide (IIIN), Type III

Oligosaccharides Coupled to Tetanus Toxoid (6-TT, 14-TT, 25-TT) or Unconjugated Tetanus Toxoid (TT)

\begin{tabular}{|c|c|c|c|c|c|}
\hline \multirow[b]{2}{*}{ Vaccine* } & \multicolumn{5}{|c|}{ Geometric mean titer (range) of serum obtained on day: } \\
\hline & 0 & 20 & 34 & 55 & 75 \\
\hline \multirow[t]{2}{*}{ IIIN } & $100^{8}$ & 100 & 126 & 100 & 100 \\
\hline & $(100)$ & $(100)$ & $(100-200)$ & $(100)$ & $(100)$ \\
\hline \multirow[t]{2}{*}{ 6-TT } & 100 & 200 & 2,340 & 16,000 & $12,700^{\prime \prime}$ \\
\hline & $(100)$ & $(100-400)$ & $(400-8,000)$ & $(16,000-16,000)$ & $(8,000-16,000)$ \\
\hline \multirow[t]{2}{*}{$14-\mathrm{TT}^{\ddagger}$} & 100 & 400 & 6,340 & 25,400 & $25,400^{\prime \prime}$ \\
\hline & $(100)$ & $(100-800)$ & $(2,000-16,000)$ & $(8,000-64,000)$ & $(8,000-64,000)$ \\
\hline \multirow[t]{2}{*}{ 25-TT } & 126 & 252 & 2,250 & 20,160 & $40,300^{\prime \prime}$ \\
\hline & $(100-200)$ & $(100-400)$ & $(1,000-8,000)$ & $(8,000-64,000)$ & $(8,000-128,000)$ \\
\hline \multirow[t]{2}{*}{ TT } & 100 & 100 & 100 & 159 & $141^{\prime}$ \\
\hline & $(100)$ & $(100)$ & $(100)$ & $(100-200)$ & $(100-200)$ \\
\hline
\end{tabular}

* Three rabbits were vaccinated with each vaccine. ${ }^{\ddagger}$ These data have been published (7) and are presented here for comparison. Antisera elicited by the three vaccines were tested concurrently. ${ }^{8}$ Titers of 100 or less are reported as 100 . " $P=0.018$ for comparison with IIIN and TT (day 75 titers). 'One rabbit died.

oligosaccharide conjugates (Table II). Each of the two subsequent vaccinations (on days 20 and 41 ) was followed, in all cases, by a brisk rise in titer, consistent with the booster response characteristic of T cell-dependent immunogens (Table II). Mean endpoint titers in rabbits vaccinated with 6-TT, 14TT, and 25-TT were $16,000,25,400$, and 40,300 , respectively (Table II). Mean endpoint titers for all three conjugate groups were significantly higher (two-sided $P$ value $=0.018$ ) than those for the control groups (IIIN and TT) but did not differ significantly from one another (two-sided $P$ value $=0.262$ ). Pooled rabbit serum raised to each of the three oligosaccharide conjugates had identical type III-specific, IgG titer of 12,800 as measured by ELISA.

ELISA inhibition studies. To assess the epitope specificity of vaccine-induced antibodies, type III oligosaccharides (6 ru, $14 \mathrm{ru}$, and $25 \mathrm{ru}$ ) and native polysaccharide were used to inhibit binding of vaccine-induced antibodies to ELISA wells coated with native GBS type III polysaccharide. Competition binding curves were generated using varying concentrations of each inhibitor (Fig. 2). The relative functional affinity of the interaction of an antiserum with short- versus long-chain saccharides (of the same repeating structure) may be considered an index of the specificity of the antibodies for a conformational epitope preferentially expressed on higher-molecularweight saccharides. The relative functional affinity of each antiserum for different-chain-length oligosaccharides and for the native polysaccharide was assessed by measurement of the $\mathrm{IC}_{50}$. As we have shown previously for antibodies raised against whole type III GBS organisms, antibodies elicited by all three oligosaccharide conjugates bound with higher affinity to longer-chain-length saccharides, as reflected by inhibition curves shifted progressively to the left. That is, the antibodies recognized not simply a particular oligosaccharide epitope, but also a specific saccharide conformation that was more fully expressed in longer-chain-length saccharides. To assess the relative specificity of each antiserum for the conformational epitope, we determined a ratio of relative binding affinity for short- versus long-chain inhibitors by dividing the $\mathrm{IC}_{50}$ of the smallest inhibitor $(6 \mathrm{ru})$ by the $\mathrm{IC}_{50}$ of the largest inhibitor (native type III polysaccharide). Although there was overlap between the $95 \%$ confidence intervals around the ratios for the three antisera, the $\mathrm{IC}_{50}$ ratios followed the same trend as the chain length of the oligosaccharide conjugate: 116,300 , and 412 for antiserum to 6-TT, 14-TT, and 25-TT, respectively

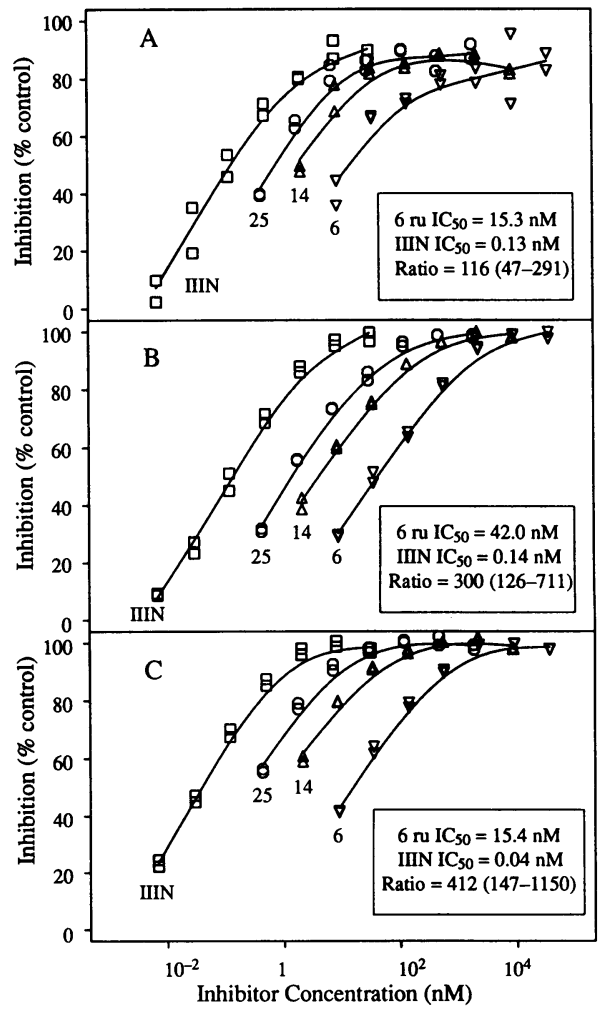

Figure 2. Group B Streptococcus type III capsular polysaccharide competitive ELISA. Data points represent the inhibition of binding to native type III polysaccharide by rabbit antibodies to 6-TT vaccine $(A)$, 14-TT vaccine $(B)$, or 25 -TT vaccine $(C)$ by type III oligosaccharides of 6-ru $(\nabla), 14-\mathrm{ru}(\Delta)$, or 25-ru (O) or by native type III polysaccharide ( $\square$ ). The $\mathrm{IC}_{50}$ ratio (and $95 \%$ confidence interval) was calculated as the ratio of $50 \%$ inhibitory concentrations of the smallest $(6$ ru) and largest (IIIN) inhibitors for each antiserum. 
(Fig. 2). In other words, the relative specificity of the antisera for long versus short chain saccharides increased with increasing chain length of the conjugate immunogen.

Opsonic activity of vaccine-induced antibodies. Whole immune rabbit serum (day 75) and isolated IgG and IgM antibodies were tested for opsonic activity against GBS type III organisms. At a 100 -fold dilution, whole rabbit antiserum to each of the three oligosaccharide vaccines resulted in killing of $\geq 88 \%$ of GBS cells by human peripheral-blood leukocytes (Table III). However, at a 400-fold dilution, rabbit antiserum to 14-TT consistently promoted $>90 \%$ killing of GBS cells, while antiserum raised to 6-TT or 25-TT showed little or no activity (Table III). IgG, separated from whole immune rabbit sera to each of the oligosaccharide conjugates, mediated $\geq 72 \%$ opsonic killing of GBS (Table IV). When diluted 400-fold, IgG from rabbits that received 14-TT vaccine still promoted killing of $25 \%$ of GBS cells. The level of opsonic killing by rabbit IgM rose in direct correlation with the chain length of the type III oligosaccharide used in the conjugate. IgM from rabbits vaccinated with 6-TT, 14-TT, or 25-TT mediated the killing of 26, 42 , and $78 \%$ of GBS cells, respectively (Table IV). When tested at a dilution of $1: 100$, antiserum to uncoupled type III polysaccharide, antiserum to unconjugated TT, and prevaccination rabbit serum all failed to promote the killing of GBS cells (not shown).

Passive protection of mice by rabbit antisera to oligosaccharide-TT conjugates. To ascertain the functional activity in vivo of immune rabbit serum to the oligosaccharide vaccines, outbred mice were injected with postvaccination rabbit serum $24 \mathrm{~h}$ before challenge with a lethal dose of viable GBS strain M781 cells. Of 16 mice that received serum from rabbits vaccinated with 6-TT conjugate, nine (56\%) survived GBS challenge, of 16 mice that received rabbit antiserum to 25-TT, five (31\%) survived (Table V). Rabbit antiserum to 14-TT conferred $100 \%$ protection against a lethal GBS challenge (7). Antiserum to the 14-TT vaccine was significantly superior to antibodies to either the 6-TT $(P=0.007)$ or the 25-TT $(P=0.0002)$ vaccine in conferring protection to mice. Therefore, as the oligosaccharide chain length used in the vaccine increased from 6

Table III. Opsonophagocytic Killing Activity of Serum (Day 75) from Rabbits Vaccinated with GBS Type III Oligosaccharide Conjugates (6-TT, 14-TT, 25-TT)

\begin{tabular}{clc}
\hline Vaccine* & $\begin{array}{c}\text { Antibody } \\
\text { dilution }\end{array}$ & $\begin{array}{c}\text { Mean percent kill } \\
\text { (Exp. 1; Exp. 2) }\end{array}$ \\
\hline \multirow{2}{*}{ 6-TT } & $1 / 100$ & $96(91,97 ; 98,99)$ \\
& $1 / 400$ & $33(0, \mp 0 ; 58,72)$ \\
$14-$ TT $^{\S}$ & $1 / 100$ & $99(99,99 ; 98,98)$ \\
& $1 / 400$ & $94(93,93 ; 94,96)$ \\
& $1 / 1600$ & $0(0,0 ; 0,0)$ \\
$25-T T$ & $1 / 100$ & $88(99,99 ; 59,95)$ \\
& $1 / 400$ & $0(0,0 ; 0,0)$
\end{tabular}

\footnotetext{
* When tested at a dilution of 1:100, antiserum to uncoupled type III polysaccharide, antiserum to unconjugated TT, and prevaccination rabbit serum all failed to promote the killing of GBS cells (not shown). ₹ A value of zero indicates growth of GBS.

Some values obtained using antiserum raised in rabbits to this vaccine have been published (7) and are presented here for comparison. Antisera elicited by the three vaccines were tested concurrently.
}

Table IV. Opsonophagocytic Killing Activity of Immunoglobulin Fractions from Rabbits Vaccinated with GBS

Type III Oligosaccharide Conjugates (6-TT, 14-TT, 25-TT)

\begin{tabular}{cccc}
\hline & \multicolumn{2}{c}{$\begin{array}{c}\text { Antibody } \\
\text { dilution }\end{array}$} & \multicolumn{2}{c}{ IgG* } & IgM $^{*}$ \\
\cline { 3 - 4 } 6-TT & $1 / 100$ & $72(72,72)$ & $26(22,30)$ \\
& $1 / 400$ & $21(0, \pm 42)$ & $-\$$ \\
14-TT" & $1 / 100$ & $96(94,97)$ & $42(37,46)$ \\
& $1 / 400$ & $25(14,35)$ & - \\
& $1 / 1600$ & $3(0,6)$ & - \\
$25-T T$ & $1 / 100$ & $77(85,68)$ & $78(76,80)$ \\
& $1 / 400$ & $13(0,25)$ & -
\end{tabular}

* IgG and IgM antibody fractions were separated by affinity chromatography on a column of protein A-agarose.

${ }^{\ddagger} \mathrm{A}$ value of zero indicates growth of GBS.

Not tested.

"Values obtained using antiserum raised in rabbits to this vaccine have been published (7) and are presented here for comparison. Antisera elicited by the three vaccines were tested concurrently.

ru to $14 \mathrm{ru}$, the protective activity of immune rabbit serum increased. However, a further increase in conjugate oligosaccharide chain length from 14 ru to 25 ru produced antiserum with diminished functional activity of the vaccine-induced antibodies.

\section{Discussion}

Capsular polysaccharides of bacterial pathogens such as Neisseria meningitidis, Streptococcus pneumoniae, Haemophilus influenzae type b, and group B Streptococcus are outer surface polymers of regularly repeating saccharide units. In many cases, bacterial capsular polysaccharides have been shown to be important targets for protective antibodies. In general, polysaccharides are considered $\mathrm{T}$ cell-independent antigens, and, as

Table V. Passive Protection of Mice by Vaccine-induced Antisera

\begin{tabular}{lrrrr}
\hline \multirow{2}{*}{\multicolumn{1}{c}{ Vaccine }} & \multicolumn{2}{c}{ No. mice survived/No. mice challenged } & \\
\cline { 2 - 3 } & Day 1 & \multicolumn{1}{c}{ Day 2 } & Day 3 & $\begin{array}{r}\text { Percent } \\
\text { survival }\end{array}$ \\
\hline 6-TT & $13 / 16$ & $9 / 16$ & $9 / 16$ & $56^{\ddagger}$ \\
14-TT* & $16 / 16$ & $16 / 16$ & $16 / 16$ & 100 \\
25-TT & $10 / 16$ & $6 / 16$ & $5 / 16$ & $31^{\S}$ \\
III native* & $1 / 17$ & $0 / 17$ & $0 / 17$ & 0 \\
Preimmunization & $9 / 17$ & $1 / 17$ & $0 / 17$ & 0
\end{tabular}

Mice received pooled antiserum of rabbits vaccinated with oligosaccharide-tetanus toxoid conjugate vaccines (6-TT, 14-TT and 25-TT) or native type III polysaccharide (III native) $24 \mathrm{~h}$ before challenge with a lethal dose of type III group B Streptococcus. Preimmunization rabbit serum was obtained on day 0 .

* These data have been published (7) and are presented here for comparison. Antisera elicited by the three vaccines were tested concurrently.

${ }^{\ddagger} P=0.007$ for 6 -TT vs. 14 -TT.

${ }^{8} P=0.0002$ for $25-\mathrm{TT}$ vs. $14-\mathrm{TT}$. 
such, they are relatively weak immunogens that elicit mainly IgM antibodies in mice. The immunogenicity of several bacterial polysaccharides or of derivative oligosaccharides has been increased by coupling to a suitable protein carrier. However, conjugation of an oligosaccharide to a protein does not insure an optimal saccharide-specific antibody response. Specific features of the conjugate design that may influence immunogenicity include choice of carrier protein, degree of carbohydrate loading, coupling method (random site or multisite versus single-site coupling), and chemical treatments that may alter the native epitope. In addition, the molecular size of the saccharide used in such a conjugate may be an important factor in the degree to which coupling to protein enhances immunogenicity. In this study we have attempted to define the effect of saccharide size on immunogenicity, using model conjugates in which other design parameters have been carefully controlled.

Detailed studies of the effects of oligosaccharide chain length on immunogenicity have been carried out by Anderson and co-workers with oligosaccharide-protein conjugates derived from the polyribosyl-ribitol phosphate capsular polysaccharide of $H$. influenzae type b (1-4). These investigators found that conjugates containing a saccharide corresponding to 20 ribose-ribitol phosphate repeating units was better able to prime human infants for a secondary response than was an 8-repeating-unit conjugate. However, in a subsequent study of oligosaccharide conjugates of a slightly different design, no difference was found in antibody responses to 4-, 6-, or 12-repeating-unit conjugates (4). This finding underscores the importance of the precise design of the conjugate in determining immune response.

Studies of dextran conjugates have provided evidence that, as part of a conjugate vaccine, shorter chain length oligosaccharides elicit a greater saccharide-specific immune response than conjugates of longer chain lengths. In $\mathrm{BALB} / \mathrm{c}$ mice, dextran conjugates of 1,000 or $10,000 \mathrm{D}$ elicited a 10 -fold greater antidextran response than conjugates containing larger dextrans of $5 \times 10^{6}$ to $40 \times 10^{6} \mathrm{D}(6)$. Seppälä and Mäkelä (10) noted that shorter chain length dextrans $\left(M_{\mathrm{r}}<7,000\right)$ coupled to proteins via their reducing ends by reductive amination elicited a better IgG class secondary response in mice than did conjugates prepared by a random-site coupling method.

While shorter-chain-length conjugates may enhance T celldependent properties of the immune response, larger saccharide haptens may better express the epitope(s) of the native polysaccharide. In studies of Salmonella typhimurium O-antigen saccharide-protein conjugates, Svenson and Lindberg (8) found a correlation between the relative specificity of the vaccine-induced antibodies for oligosaccharides of different sizes, and the size of the oligosaccharide used in the immunizing conjugate. Although their studies examined a rather narrow range of saccharide chain lengths (1-3 tetrasaccharide repeating units), these investigators concluded that "larger saccharide haptens elicit antibody populations which recognize the native bacterial polysaccharide better than antibodies evoked by smaller saccharide lengths" (8). These and other studies $(1,22)$ suggest that the oligosaccharide portion of the conjugate ideally should be small enough to function as a $\mathrm{T}$ cell-dependent hapten, yet large enough to have antigenically complete epitopes like those found on the native polysaccharide (8). In addition the haptenization method used to prepare the oligosaccharide conjugate vaccines should not alter critical antigenic epitopes.

These immunological considerations guided the design of
GBS type III oligosaccharide vaccines. An important element in the epitope of the GBS type III polysaccharide is the acid-labile sialic acid residues present as side chain termini $(23,24)$. To maintain the structural and chemical integrity of derivative oligosaccharides, GBS type III oligosaccharides with intact sialic acid residues were prepared with endo- $\beta$-galactosidase (25). Because endo- $\beta$-galactosidase cleaves internal $\beta(1 \rightarrow 4)$ galactosidic linkages found in the backbone of type III polysaccharide $(7,26)$, we were able to generate and purify GBS type III oligosaccharides of varying chain lengths, with an intact repeating-unit structure, for conjugation to tetanus toxoid. The conjugation method allowed well-defined, single-site covalent attachment of oligosaccharides of three different chain lengths to TT. The three oligosaccharide conjugates had approximately the same molar ratio of oligosaccharide to protein; thus the effects of the various saccharide chain lengths on immunogenicity could be compared directly. Immunogenicity (as determined by ELISA) of GBS type III polysaccharide was dramatically enhanced by coupling of type III oligosaccharides to TT. Increased immune response was observed regardless of the oligosaccharide chain length used in the vaccines. However, when tested for the ability to mediate killing of GBS cells by human blood leukocytes in vitro or to provide in vivo protection against a lethal GBS challenge, rabbit antiserum to the 14-TT vaccine was consistently superior to antisera to the other vaccines. Thus, anticapsular antibody titers determined by ELISA may not be a precise predictor of the functional capacity of antibodies to GBS type III.

There was a trend toward increased killing of GBS mediated by IgM antibodies as the size of the oligosaccharide used in the vaccine increased: IgM antibodies elicited by 6-TT vaccine mediated $26 \%$ killing in opsonophagocytic assays, whereas IgM antibodies elicited by $25-\mathrm{TT}$ mediated $78 \%$ killing. This trend is consistent with a shift from a $\mathrm{T}$ cell-dependent response (characterized by high IgG and low IgM opsonic titers) elicited by the 6-TT and 14-TT vaccines to a response characteristic of a T cell-independent antigen (with higher IgM and relatively less IgG opsonic titers) elicited by 25 -TT vaccine. Because these oligosaccharides were coupled at a single site through the end of the saccharide chain, the conformational epitope of 25-TT may have been sufficiently separated from the protein moiety that carrier-specific $T$ cells had less influence than in shorter-chain-length conjugates on modification of the saccharide-specific B-cell response.

We also examined epitope specificity of oligosaccharide conjugate-induced antibodies. Antibodies raised in rabbits against whole GBS type III organisms bind with much higher affinity to high-molecular-weight forms of the type III polysaccharide than to short-chain-length oligosaccharides of the same repeating structure (13). We have proposed that the relative specificity of these antibodies for a conformational epitope expressed only in high-molecular-weight saccharides may represent a mechanism by which the host immune response discriminates between this (and other) polysaccharide antigens and host oligosaccharides of similar structure $(13,14)$. It is not surprising, then, that the largest oligosaccharide conjugate (25TT) elicited antibodies that bound with $\sim 400$-fold higher functional affinity to the native polysaccharide than to a 6-ru oligosaccharide. The difference in relative binding affinity of the 14-TT-induced antibodies to these two antigens was 300fold; for 6-TT-induced antibodies, it was $\sim 100$-fold. These data are consistent with the hypothesis that longer-chain- 
length saccharides better express a conformational epitope important not only in antibody binding but also in the specificity of antibodies induced by saccharide conjugates of different chain lengths. The loss of conformational epitope specificity may explain the diminished ability of the 6-TT vaccine than of the larger saccharide conjugates to elicit polysaccharide-specific protective antibodies.

Our studies showed an intermediate-chain-length $(14,500$ $M_{\mathrm{r}}$ ) type III oligosaccharide conjugate to be an immunogen superior to either smaller or larger saccharide conjugates. Increased or decreased saccharide size was associated with diminished functional activity of vaccine-induced antisera. The reduction in immunogenicity of the longer-chain-length conjugate was accompanied by a shift to proportionately greater IgM response, compatible with the immune response expected for a more $T$ cell-independent immunogen. The reduced immunogenicity of the smaller conjugate suggests that the 6-ru oligosaccharide does not adequately express the conformational epitope of the native polysaccharide, a conclusion supported by the results of epitope specificity studies of the vaccine-induced antibodies. The saccharide chain length giving the best antibody response in our studies is somewhat larger than that of the dextran oligosaccharides found by Mäkelä et al. (6) to be optimal for experimental conjugate vaccines. The difference between the two systems may reflect the importance of the molecular size-dependent conformational epitope of the GBS type III polysaccharide. Our results indicate that the characteristics of the immune response to synthetic vaccines based on this bacterial polysaccharide antigen-perhaps on others as well-cannot necessarily be predicted by studies of simpler oligosaccharides derived from simpler linear polysaccharides. The presence of conformational epitopes on a variety of bacterial polysaccharides may be an important determinant of the optimal size of the derivative oligosaccharides used in conjugate vaccines against organisms bearing polysaccharide surface antigens.

\section{Acknowledgments}

We thank April Blodgett, Tom DiCesare, and Jason Berry for expert technical assistance.

This work was supported by National Institutes of Health grants AI23339, AI28040, and CA50597 and by a Grant-in-Aid from the American Heart Association. J. DiFabio and F. Michon were supported by IAF Biochem Int., Laval, P. Q., Canada. L. C. Paoletti was the recipient of Individual National Research Service Award 1F32AI08222-01.

\section{References}

1. Anderson, P. 1983. Antibody responses to Haemophilus influenzae type b and diphtheria toxin induced by conjugates of oligosaccharides of the type $b$ capsule with the nontoxic protein CRM197. Infect. Immun. 39:233-238.

2. Anderson, P., M. E. Pichichero, and R. A. Insel. 1985. Immunogens consisting of oligosaccharides from the capsule of Haemophilus influenzae type b coupled to diphtheria toxoid or CRM197. J. Clin. Invest. 76:52-59.

3. Anderson, P. W., M. E. Pichichero, R. A. Insel, R. Betts, R. Eby and D. H. Smith. 1986. Vaccines consisting of periodate-cleaved oligosaccharides from the capsule of Haemophilus influenzae type b coupled to a protein carrier: structural and temporal requirements for priming in the human infant. J. Immunol. 137:1181-1186.

4. Anderson, P. W., M. E. Pichichero, E. C. Stein, S. Porcelli, R. F. Betts,
D. M. Connuck, D. Korones, R. A. Insel, J. M. Zahradnik, and R. Eby. 1989. Effect of oligosaccharide chain length, exposed terminal group, and hapten loading on the antibody response of human adults and infants to vaccines of Haemophilus influenzae type b capsular antigen uniterminally coupled to the diphtheria protein crm 197. J. Immunol. 142:2464-2468.

5. Avery, O. T., and W. F. Goebel. 1931. Chemo-immunological studies on conjugated carbohydrate-proteins. V. The immunological specificity of an antigen prepared by combining the capsular polysaccharide of type III pneumococcus with foreign protein. J. Exp. Med. 54:437-447.

6. Mäkelä, O., F. Peterfy, I. G. Outschoorn, A. W. Richter, and I. Seppälä. 1984. Immunogenic properties of alpha $(1 \rightarrow 6)$ dextran, its protein conjugates, and conjugates of its breakdown products in mice. Scand. J. Immunol. 19:541550.

7. Paoletti, L. C., D. L. Kasper, F. Michon, J. DiFabio, K. Holme, H. J. Jennings, and M. R. Wessels. 1990. An oligosaccharide-tetanus toxoid conjugate vaccine against type III group B Streptococcus. J. Biol. Chem. 265:18278-18283.

8. Svenson, S. B., and A. A. Lindberg. 1983. Artificial Salmonella vaccines. Prog. Allergy. 33:120-143.

9. Wessels, M. R., L. C. Paoletti, D. L. Kasper, J. L. DiFabio, F. Michon, K. Holme, and H. J. Jennings. 1990. Immunogenicity in animals of a polysaccharide-protein conjugate vaccine against type III group B Streptococcus. J. Clin. Invest. 86:1428-1433.

10. Seppälä, I., and O. Mäkelä. 1989. Antigenicity of dextran-protein conjugates in mice. Effect of molecular weight of the carbohydrate and comparison of two modes of coupling. J. Immunol. 143:1259-1264.

11. Jennings, H. J., R. Roy, and F. Michon. 1985. Determinant specificities of the groups $\mathrm{B}$ and $\mathrm{C}$ polysaccharides of Neiserria meningitidis. J. Immunol. 134:2651-2657.

12. Michon, F., J. R. Brisson, and H. J. Jennings. 1987. Conformational differences between linear alpha $(2 \rightarrow 8)$-linked homosialooligosaccharides and the epitope of the group B meningococcal polysaccharide. Biochemistry. 26:8399-8405.

13. Wessels, M. R., A. Muñoz, and D. L. Kasper. 1987. A model of high-affinity antibody binding to type III group B Streptococcus capsular polysaccharide. Proc. Natl. Acad. Sci. USA. 84:9170-9174.

14. Wessels, M. R., and D. L. Kasper. 1989. Antibody recognition of the type 14 pneumococcal capsule. J. Exp. Med. 169:2121-2131.

15. Kabat, E. A., J. Liao, E. F. Osserman, A. Gamian, F. Michon, and H. J. Jennings. 1988. The epitope associated with the binding of the capsular polysaccharide of the group B meningococcus and of Escherichia coli K1 to a human monoclonal macroglobulin, IgM ${ }^{\text {NOv }}$. J. Exp. Med. 168:699-711.

16. Warren, L. 1959. The thiobarbituric acid assay of sialic acids. J. Biol. Chem. 234:1971-1975.

17. Dubois, M., K. A. Gilles, J. K. Hamilton, P. A. Rebers, and F. Smith. 1956. Colorimetric method for the determination of sugars and related substances. Anal. Chem. 28:350-356.

18. Lowry, O. H., N. J. Rosebrough, A. L. Farr, and R. J. Randall. 1951. Protein measurement with the Folin phenol reagent. J. Biol. Chem. 193:265-275.

19. Larson, E., B. Howlett, and A. Jagendorf. 1986. Artificial reductant enhancement of the Lowry method for protein determination. Anal. Biochem. 155:243-248.

20. Rubens, C. E., M. R. Wessels, L. M. Heggen, and D. L. Kasper. 1987. Transposon mutagenesis of type III group B Streptococcus: correlation of capsule expression with virulence. Proc. Natl. Acad. Sci. USA. 84:7208-7212.

21. Baltimore, R. S., D. L. Kasper, C. J. Baker, and D. K. Goroff. 1977. Antigenic specificity of opsonophagocytic antibodies in rabbit anti-sera to group B streptococci. J. Immunol. 118:673-678.

22. Seppälä, I., H. Sarvas, O. Mäkelä, P. Mattila, J. Eskola, and H. Kayhty. 1988. Human antibody responses to two conjugate vaccines of Haemophilus influenzae type B saccharides and diphtheria toxin. Scand. J. Immunol. 28:471479.

23. Jennings, H. J., C. Lugowski, and D. L. Kasper. 1981. Conformational aspects critical to the immunospecificity of the type III group B streptococcal polysaccharide. Biochemistry. 20:4511-4518.

24. Wessels, M. R., C. E. Rubens, V.-J. Benedi, and D. L. Kasper. 1989. Definition of a bacterial virulence factor: sialylation of the group B streptococcal capsule. Proc. Natl. Acad. Sci. USA. 86:8983-8987.

25. Fukuda, M. N., and G. Matsumura. 1976. Endo- $\beta$-galactosidase of Escherichia freundii. 251:6218-6225.

26. Wessels, M. R., V. Pozsgay, D. L. Kasper, and H. J. Jennings. 1987. Structure and immunochemistry of an oligosaccharide repeating unit of the capsular polysaccharide of type III group B Streptococcus. J. Biol. Chem. 262:82628267.

27. Joint Commission on Biological Nomenclature. 1982. Abbreviated terminology of oligosaccharide chains. Recommendations 1980. Eur. J. Biochem. 126:433-437. 